\title{
Pathology and pathobiology of pulmonary hypertension: state of the art and research perspectives
}

\author{
Marc Humbert (10 ${ }^{1,2,3}$, Christophe Guignabert (10 ${ }^{1,2}$, Sébastien Bonnet ${ }^{4,5}$, \\ Peter Dorfmüller $\mathbb{1}^{1,2,6}$, James R. Klinger ${ }^{7}$, Mark R. Nicolls ${ }^{8,9,10}$, \\ Andrea J. Olschewski ${ }^{11,12}$, Soni S. Pullamsetti (13 ${ }^{13,14}$, Ralph T. Schermuly (15), \\ Kurt R. Stenmark ${ }^{16}$ and Marlene Rabinovitch ${ }^{8,9,10}$ \\ Number 1 in the series \\ "Proceedings of the 6th World Symposium on Pulmonary Hypertension" \\ Edited by N. Galiè, V.V. McLaughlin, L.J. Rubin and G. Simonneau
}

@ERSpublications

State of the art and research perspectives in the cellular and molecular basis and pathology of pulmonary vascular remodelling associated with various forms of pulmonary hypertension http://ow.ly/cjwp30mgzmH

Cite this article as: Humbert M, Guignabert C, Bonnet S, et al. Pathology and pathobiology of pulmonary hypertension: state of the art and research perspectives. Eur Respir J 2019; 53: 1801887 [https://doi.org/ $10.1183 / 13993003.01887-2018]$.

ABSTRACT Clinical and translational research has played a major role in advancing our understanding of pulmonary hypertension (PH), including pulmonary arterial hypertension and other forms of $\mathrm{PH}$ with severe vascular remodelling (e.g. chronic thromboembolic PH and pulmonary veno-occlusive disease). However, $\mathrm{PH}$ remains an incurable condition with a high mortality rate, underscoring the need for a better transfer of novel scientific knowledge into healthcare interventions. Herein, we review recent findings in pathology (with the questioning of the strict morphological categorisation of various forms of $\mathrm{PH}$ into pre- or post-capillary involvement of pulmonary vessels) and cellular mechanisms contributing to the onset and progression of pulmonary vascular remodelling associated with various forms of $\mathrm{PH}$. We also discuss ways to improve management and to support and optimise drug development in this research field. 


\section{Introduction}

Pulmonary hypertension (PH) encompasses a group of severe clinical entities, such as pulmonary arterial hypertension (PAH) and chronic thromboembolic PH (CTEPH), in which loss and obstructive remodelling of the pulmonary vascular bed is responsible for the rise in pulmonary arterial pressure and pulmonary vascular resistance (PVR), resulting in progressive right heart failure and functional decline. Pulmonary vascular remodelling in PAH is not only characterised by an accumulation of different vascular cells in the pulmonary arterial wall (pulmonary artery smooth muscle cells (PA-SMCs), endothelial cells, fibroblasts, myofibroblasts and pericytes), but also by loss of pre-capillary arteries and by an exaggerated perivascular infiltration of inflammatory cells (B- and T-lymphocytes, mast cells, dendritic cells, macrophages, etc.). Because current PAH treatments do not specifically target pulmonary vascular remodelling and inflammation, there is an urgent need to better identify the pathobiological mechanisms underlying the progressive narrowing of the pulmonary arterial lumen and perivascular inflammation and the loss of vessels in order to support therapeutic innovation aimed at reversing these features and regenerating normal pulmonary vessels.

\section{Recent advances in pathology and laboratory medicine General considerations}

In addition to stiffening of large elastic main, lobar and segmental pulmonary arteries, $\mathrm{PH}$ can be attributed to lesions mainly occurring in distal muscular-type arteries, ranging in diameter from $500 \mu \mathrm{m}$ down to $70 \mu \mathrm{m}$ in humans (medial hypertrophy/hyperplasia, intimal and adventitial fibrosis, and (in situ) thrombotic lesions, plexiform lesions) (figure 1). They can be clearly differentiated from pulmonary veins due to their topography within the lung, since they are always neighboured by an airway (bronchiole), as well as through their microscopic anatomy, which includes a neatly defined tunica media that is delimited by the internal and the external elastic lamina. Small pre-capillary pulmonary arteries ranging in diameter from $70 \mu \mathrm{m}$ down to $20 \mu \mathrm{m}$ in humans (arterioles) are also involved in all groups of human and experimental $\mathrm{PH}$, through processes of loss and obliteration, abnormal muscularisation, and perivascular inflammation (figure 2). In contrast to the muscular-type arteries, they can only be indirectly distinguished from small post-capillary venules of the same size, through serial section tracing, or, if feasible, injection techniques with dye or beads. The capillary compartment that arises from the arteriolar microvasculature and that represents the largest vascular surface within the lung is also frequently involved. There is also accumulating evidence supporting the involvement of the post-capillary pulmonary venous vasculature in all $\mathrm{PH}$ groups with varying degrees of intensity. In $\mathrm{PH}$ due to left heart disease and chronic respiratory disease, post-capillary involvement is likely to be explained at least in part by parenchymal destruction and inflammation in patients with lung fibrosis and emphysema, and by chronic elevation in post-capillary pressure in left heart failure where $\mathrm{PH}$ is associated with global pulmonary vascular remodelling. Interestingly, the severity of $\mathrm{PH}$ in heart failure correlates with venous and small indeterminate vessel intimal thickening, resembling the pattern observed in pulmonary veno-occlusive disease (PVOD) [1]; larger pulmonary veins running within the interlobular septa may appear

Affiliations: ${ }^{1}$ Faculté de Médecine, Université Paris-Sud and Université Paris-Saclay, Le Kremlin-Bicêtre, France. ${ }^{2}$ INSERM UMR_S 999, Le Plessis-Robinson, France. ${ }^{3}$ AP-HP, Service de Pneumologie, Centre de Référence de l'Hypertension Pulmonaire Sévère, Département Hospitalo-Universitaire (DHU) Thorax Innovation (TORINO), Hôpital de Bicêtre, Le Kremlin-Bicêtre, France. ${ }^{4}$ Pulmonary Hypertension Research Group, Centre de Recherche de l'Institut de Cardiologie et de Pneumologie de Quebec, Quebec City, QC, Canada. ${ }^{5}$ Dept of Medicine, Université Laval, Quebec City, QC, Canada. ${ }^{6}$ Pathology Dept, Hôpital Marie Lannelongue, Le Plessis-Robinson, France. ${ }^{7}$ Division of Pulmonary, Critical Care and Sleep Medicine, Dept of Medicine, Rhode Island Hospital, Warren Alpert Medical School of Brown University, Providence, RI, USA. ${ }^{8}$ Cardiovascular Institute, Dept of Pediatrics, Stanford University School of Medicine, Stanford, CA, USA. ${ }^{9}$ Division of Pulmonary and Critical Care Medicine, Dept of Medicine, Stanford University School of Medicine/ VA Palo Alto, Palo Alto, CA, USA. ${ }^{10}$ The Vera Moulton Wall Center for Pulmonary Vascular Disease, Stanford, CA, USA. ${ }^{11}$ Ludwig Boltzmann Institute for Lung Vascular Research, Graz, Austria. ${ }^{12}$ Institute of Physiology, Medical University of Graz, Graz, Austria. ${ }^{13}$ Max Planck Institute for Heart and Lung Research Bad Nauheim, Bad Nauheim, Germany. ${ }^{14}$ Justus-Liebig University Giessen, Excellence Cluster Cardio Pulmonary Institute (CPI), Giessen, Germany. ${ }^{15}$ University of Giessen and Marburg Lung Centre (UGMLC), Justus-Liebig University Giessen and Member of the German Center for Lung Research (DZL), Excellence Cluster Cardio Pulmonary Institute (CPI), Giessen, Germany. ${ }^{16}$ Developmental Lung Biology and Cardiovascular Pulmonary Research Laboratories, University of Colorado, Denver, CO, USA.

Correspondence: Marlene Rabinovitch, Cardiovascular Institute, Dept of Pediatrics, Stanford University School of Medicine, 269 Campus Drive, CCSR Building, Room 1215A, Stanford, CA 94305-5162, USA. E-mail: marlenerdstanford.edu

Correspondence:Marc Humbert, Université Paris-Sud, Service de Pneumologie, Hôpital Bicêtre, AP-HP, 78 rue du Général Leclerc, 94270 Le Kremlin-Bicêtre, France. E-mail: marc.humbert囚aphp.fr 

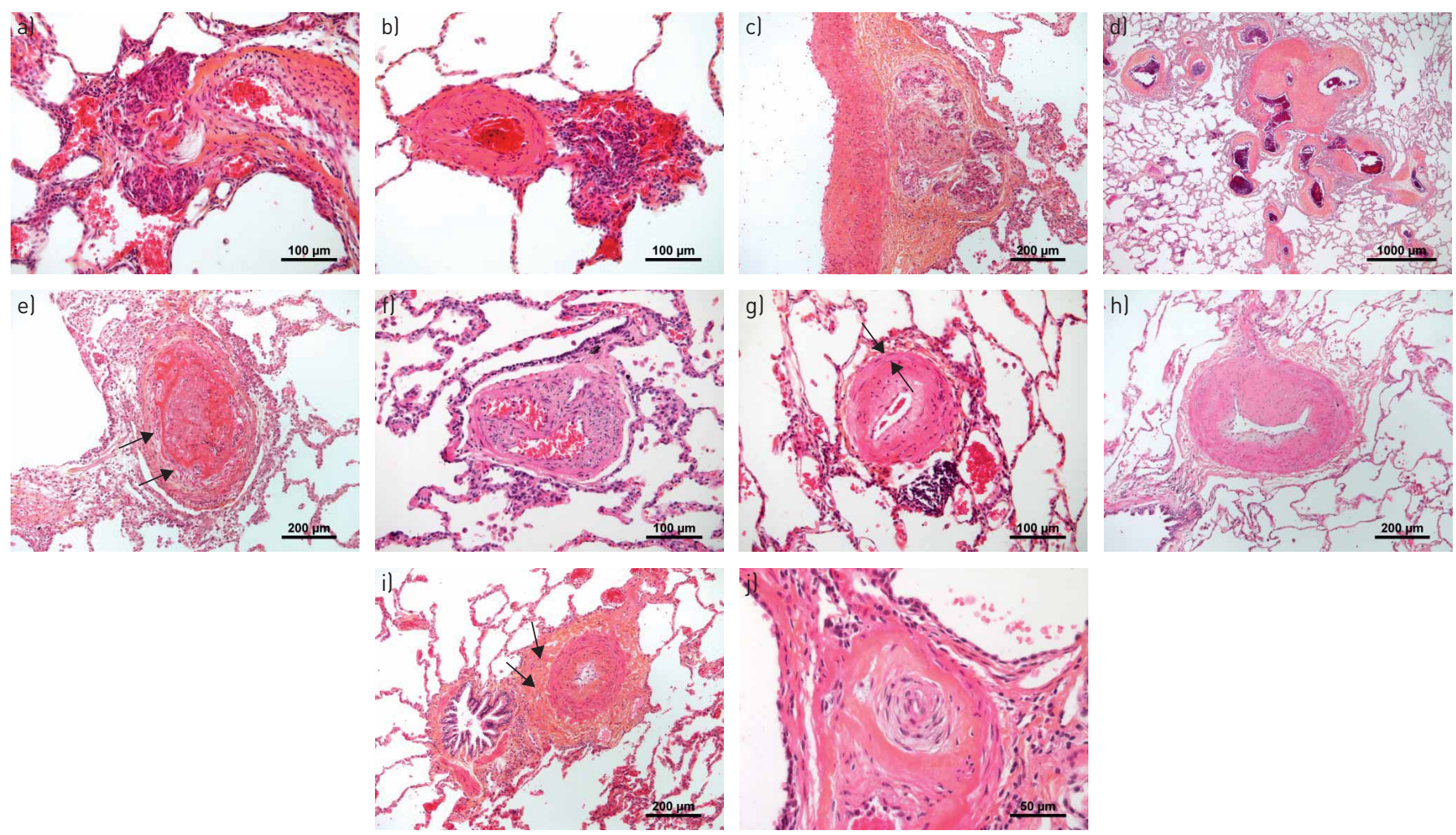

FIGURE 1 Representative vascular lesions typically detected in lungs of patients with pulmonary arterial hypertension (PAH). a-c) Plexiform lesions. Note that the plexiform core can be in $a, b)$ the para-arterial position, appearing connected to the adventitia, or c) even within the perimeters of the latter, possibly indicating an involvement of the systemic vasculature (vasa vasorum). d) Atypical fibrovascular lesions (also referred to as "SiMFis" (singular millimetric fibrovascular lesions)) of millimetric size (note the scale bar) that can be found in PAH, mostly in its hereditary bone morphogenetic protein receptor type 2-related form. e) Recent thrombotic lesion that shows fresh fibrin at its core and a beginning organising process involving numerous fibroblasts in its periphery (arrows). f) Fully organised thrombotic lesion ("colander-like lesion") with several recanalisation vessels, vaguely reminiscent of a plexiform lesion. g) Concentric, non-laminar fibrosis of the intima; the media (arrows) is only slightly thickened (note the lymphocyte-rich infiltrate in the lower periphery of the vessel). h) Eccentric, cushion-like fibrosis of the intima, commonly interpreted as an organised thrombotic lesion. i) Hyperplasia of the media and collagen-rich fibrosis of the adventitia (arrows). j) Concentric laminar fibrosis of the intima ("onion-skin lesion") due to the concentrically arranged multiple fibrous layers that lead to the progressive obstruction of the pulmonary artery.

"arteriolised", mimicking the exact microscopic anatomy of muscular-type pulmonary arteries. In apparently pure pre-capillary forms of $\mathrm{PH}$, such as $\mathrm{PAH}$ and $\mathrm{CTEPH}$, the mechanisms of post-capillary involvement are not obvious, since the post-capillary vessels, in theory, should be shielded from increased pre-capillary pressure by the arterial and capillary compartment [2].

\section{New interpretation of specific pulmonary vascular lesions \\ Plexiform lesions/complex lesions}

Complex pulmonary arterial lesions comprise different elements, such as onion-skin lesions, plexiform core lesions and dilation lesions, which are commonly observed in close topographic association (figure 1a-c). However, the pathophysiological significance of these typical vascular changes in PAH has yet to be elucidated. In this regard, recent reports suggest that systemic vessels, such as the vasa vasorum and bronchial arteries running within the adventitia of pulmonary arteries or within the peribronchial connective tissue, respectively, could be involved in the plexiform vasculopathy (figure 1c). Analysis of serial sections from PAH patients with digital three-dimensional reconstruction supports a shunting hypothesis, where plexiform lesions appear to represent anastomosing structures between bronchial microvessels and pulmonary arteries and veins [3]. Shunting between the bronchial and pulmonary vasculature has been described by means of morphometric analysis of explanted lung tissue sections from PAH patients, including idiopathic PAH (IPAH) and heritable PAH (HPAH) due to a BMPR2 (bone morphogenetic protein receptor type 2) mutation [4]. Hypertrophy and dilatation of bronchial arteries and increase in bronchial microvessel density in BMPR2 mutation carriers correlated with pulmonary venous remodelling [4]. Moreover, large fibrous vascular structures ("SiMFis" (singular millimetric fibrovascular lesions)) appear to connect the systemic vasculature to pulmonary arteries and veins (figure 1d). A 

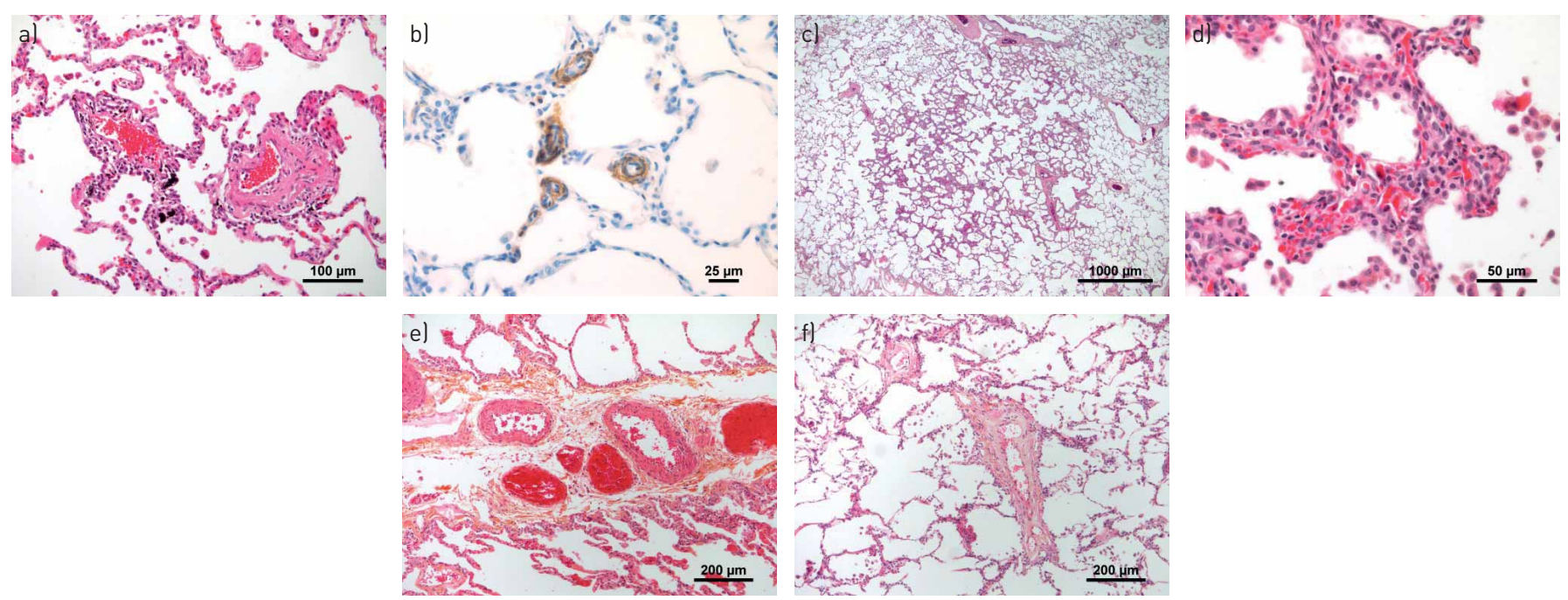

FIGURE 2 Representative vascular lesions typically detected in the microvasculature, capillaries and post-capillary vessels (pulmonary veins) in lungs of patients with pulmonary arterial hypertension (PAH) or pulmonary veno-occlusive disease (PVOD). a) Microvessels (arteries or venules) presenting some mild inflammatory and fibrous remodelling (patient with PAH). b) Representative image of $\alpha$-smooth muscle actin immunostaining in lungs of a patient with PVOD, highlighting substantial muscularisation of the pulmonary vasculature. cl Roundish, well-delimited area of interstitial thickening in a patient with PVOD; these areas are distributed in a patchy fashion throughout the lungs of patients with PVOD and probably correspond to typical ground-glass opacities on computed tomography scan. d) At higher magnification, the interstitial thickening in (c) is due to focal multiplication of alveolar capillaries that form multiple layers within the alveolar septa; the term capillary haemangiomatosis-like foci describes this patchy interstitial pattern. e) Muscular hyperplasia and fibrous remodelling in pulmonary septal veins of a patient with PAH. f) Fibrous intima thickening of small septal veins in a patient with PVOD.

functional role for the hypertrophic systemic vasculature in PAH that would allow short-circuiting a primary pulmonary arterial obstruction (figure 3 ) has yet to be confirmed.

Venous and venular lesions

A substantial proportion of PH patients display pulmonary venous and venular remodelling (figure 2e) [4]: lungs from PAH patients with scleroderma often exhibit PVOD-like pathology [5], and CTEPH lungs commonly show pulmonary veins and venules abnormalities [2]. CTEPH is of particular interest in this context. Although the primary insult, i.e. chronic thromboembolic occlusion of elastic and muscular arteries, occurs on the pre-capillary side of the pulmonary vasculature and contributes to increased PVR, remodelling of microvessels is also present, affecting pre-capillary arterioles and post-capillary venules [2, 6]. Importantly, bronchial arterial hypertrophy is associated with pulmonary venous remodelling in CTEPH, supporting the concept that systemic lung vessels associated with bronchopulmonary anastomoses could contribute to these changes [2].

In PVOD, pulmonary vascular lesions are thought to predominate on the post-capillary side, but arteries are also involved [7]. Post-capillary lesions affecting septal veins and pre-septal venules frequently consist of loose, fibrous remodelling of the intima that may totally occlude the lumen. The walls of septal veins and pre-septal venules may show smooth muscle cell hyperplasia and can be difficult to distinguish from abnormally muscularised arterioles $<70 \mu \mathrm{m}$ in diameter in PVOD lungs [7]. Post-capillary remodelling is frequently associated with pulmonary capillary angioectasia and capillary angioproliferation with doubling and tripling of the alveolar septal capillary layers that may be focally distributed (pulmonary capillary haemangiomatosis). See figure $2 \mathrm{~b}-\mathrm{d}$ and $\mathrm{f}$.

\section{Recent advances in cellular abnormalities and emerging therapeutic targets Dysfunction of pulmonary vascular endothelium}

In $\mathrm{PAH}$, the term pulmonary endothelial dysfunction has been used to denote impairment of endothelial-dependent vasodilatation in favour of vasoconstriction, but it also refers to reduced anticoagulant properties, active metabolic changes, reactive oxygen species production, increased expression of adhesion molecules (E-selectin, intercellular adhesion molecule 1 (ICAM1) and vascular cell adhesion molecule 1 (VCAM1)), and a local unadapted release of different chemokines, cytokines and growth factors (figure 4). These latter changes result in impairments in angiogenesis and repair mechanisms that play primary roles in pulmonary vascular remodelling [8]. It is now well established that cultured pulmonary endothelial cells from patients with PAH maintain in vitro several abnormal phenotypic features more or less pronounced, perhaps reflecting different subpopulations. Among them, 


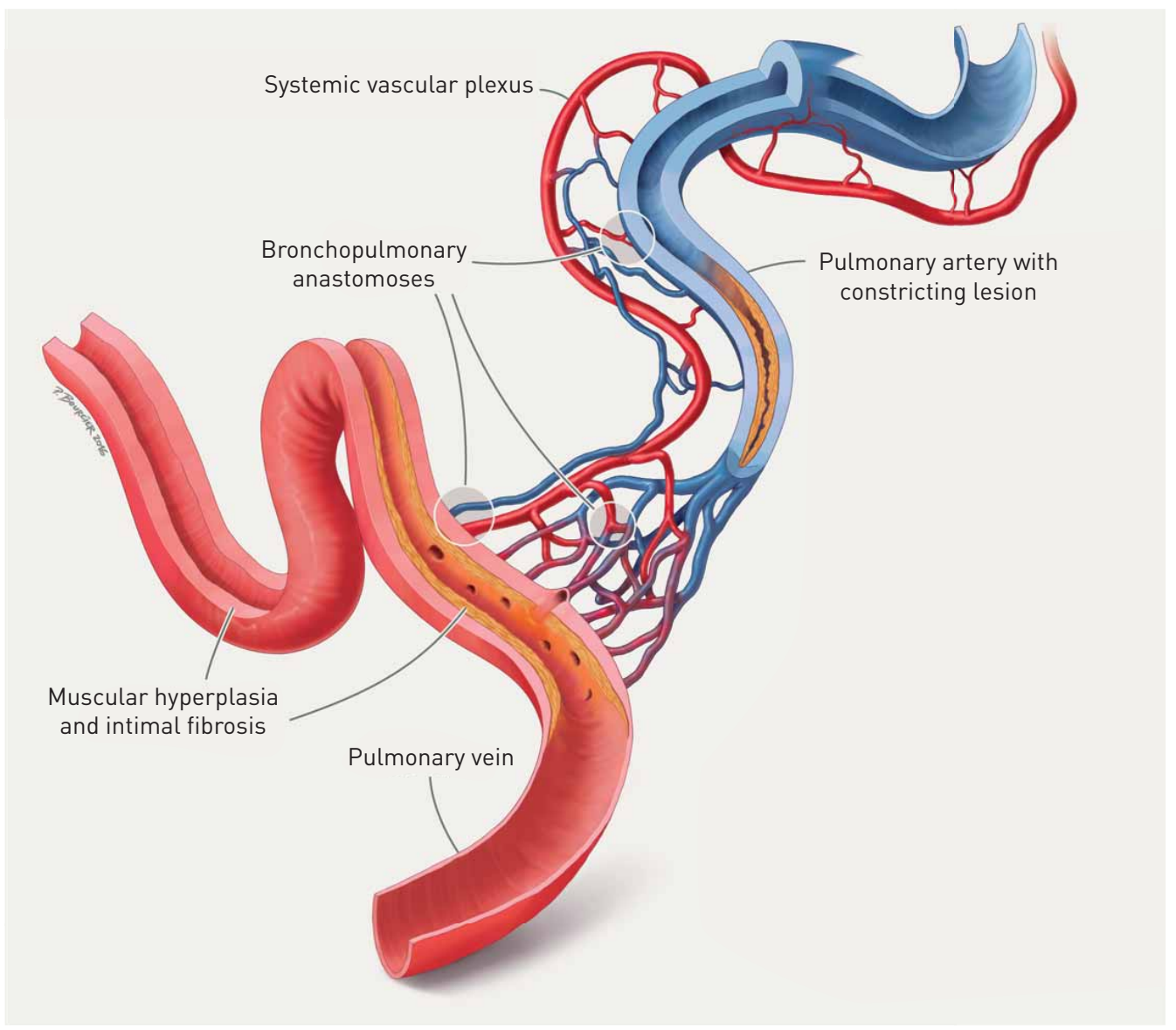

FIGURE 3 Impact of hypertrophic systemic vasculature in pulmonary arterial hypertension (PAH): an explanatory approach. The pulmonary artery (top centre, blue) is covered by a systemic vascular plexus, comprising systemic arterial (red) and venous (blue) vessels and microvessels. The systemic plexus anastomoses with the pulmonary artery, the capillary bed and the pulmonary vein (bottom left, red): these bronchopulmonary anastomoses appear to bypass an occlusive PAH lesion, represented by medial thickening and intimal fibrosis (centre). Eventually, the increased systemic blood flow into arterioles, capillaries and the pulmonary vein leads to structural changes of the latter: muscular hyperplasia and focal intimal fibrosis within the pulmonary vein are observed. Reproduced and modified from [4] with permission.

decreased capacity for vascular tube formation in vitro, heightened aerobic glycolysis, and loss of some endothelial cell markers and acquisition of several mesenchymal cell markers have been described [9-11]. In $\mathrm{PAH}$, a pro-inflammatory phenotype of pulmonary endothelial cells characterised by an increase in surface expression of E-selectin, ICAM1 and VCAM1 together with an excessive release of various key cytokines and chemokines has been reported [12]. Some features can be reproduced in endothelial cells grown from induced pluripotent stem cells (iPSCs) derived from the skin of the same patients [13, 14]. Various stimuli, such as high glucose, insulin resistance, disturbed blood flow and oxidative stress, can lead to endothelial dysfunction. However, the cause and the underlying mechanisms responsible for dysfunction of the pulmonary endothelium in PAH are still incompletely understood.

Recent studies have focused on two key modulators of endothelial structure and function that initiate and perpetuate pulmonary vascular remodelling associated with $\mathrm{PH} / \mathrm{PAH}$, i.e. fluid flow-induced high shear stress as well as low oxygen tension (chronic hypoxia). Normally, vascular endothelial cells respond to high shear stress by losing their cobblestone appearance and elongating in the direction of flow. Failure to adapt these morphological changes is associated with an increased tendency toward vascular remodelling. Interestingly, pressure off-loading by pulmonary artery banding has been reported to prevent and even reverse occlusive vascular remodelling in the Sugen hypoxia rat model [15]. This observation is consistent with findings from more recent studies showing that microvascular pulmonary endothelial cells, but not proximal pulmonary artery endothelial cells, isolated from PAH patients exhibit delayed morphological adaptation to high shear stress in vitro [16]. Chronic hypoxia also gives rise to structural remodelling of the pulmonary vasculature in experimental and human PH. Recent studies have supported this notion by showing that pulmonary vascular endothelial cells from plexiform lesions in patients with PAH have 


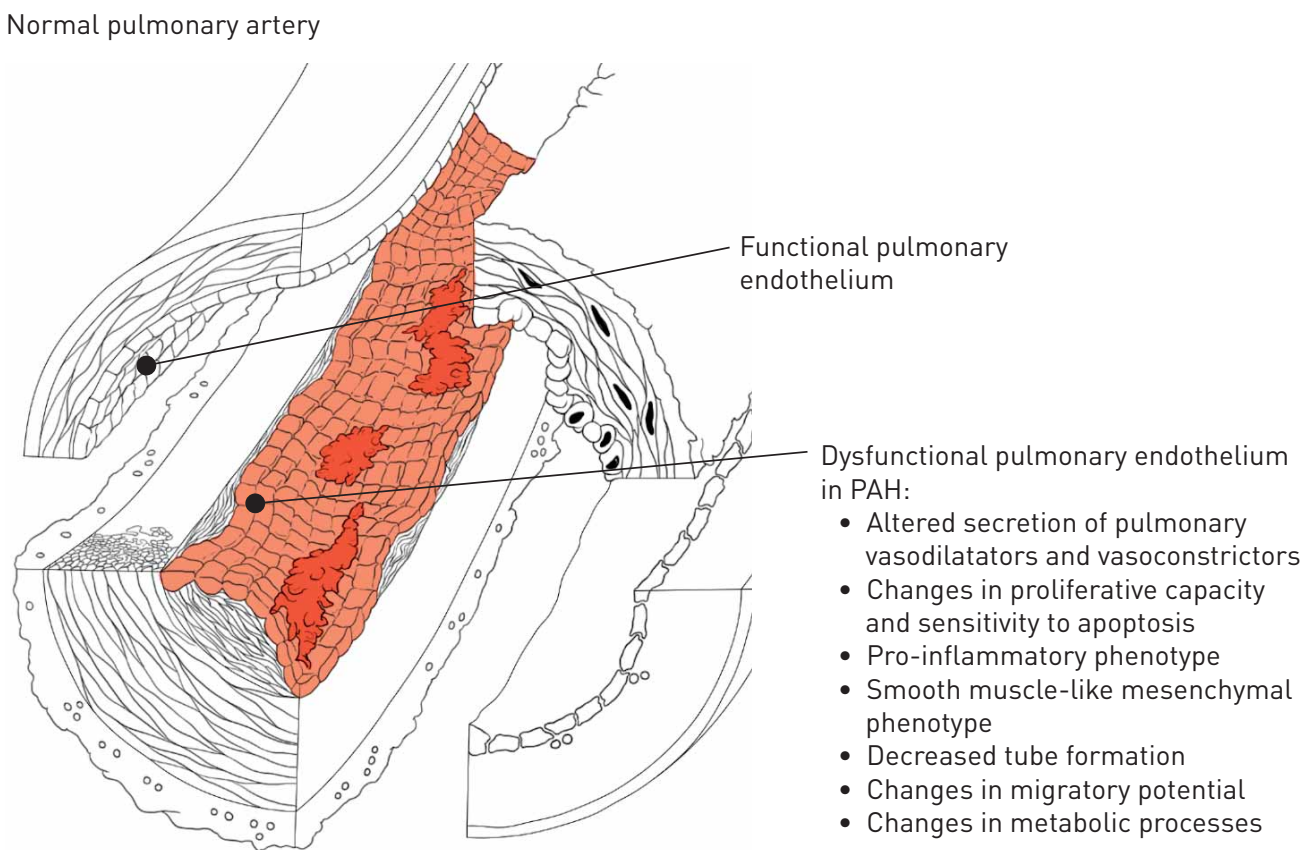

PAH pulmonary artery

FIGURE 4 Phenotypic signature of dysfunctional pulmonary vascular endothelium in pulmonary arterial hypertension (PAH).

decreased expression of prolyl-4 hydroxylase 2 (PHD2), an enzyme that facilitates the degradation of the hypoxic-inducible factor HIF1 and HIF2 hypoxia sensors. Furthermore, mice with endothelial cell-targeted disruption of the gene for PHD2 (EGLN1) develop obliterative pulmonary vascular remodelling and complex lesions as found in human $\mathrm{PAH}$ [17]. A combination of amphetamine and hypoxia can increase endothelial propensity to apoptosis and cause DNA damage by interfering with the normal metabolic function of HIF1 $\alpha$ in switching to a glycolytic state [18]. Dasatinib can induce PAH and has also emerged as a modulator of pulmonary endothelial function: dasatinib at high doses induces pulmonary endothelial cell dysfunction via increased production of reactive oxygen species, thereby increasing the susceptibility to pleural effusions and $\mathrm{PH}$ in rodents [19-21]. A better understanding of the molecular mechanisms underlying endothelial adaptation to high shear stress and chronic hypoxia will greatly enhance our understanding of the pathogenesis of $\mathrm{PH} / \mathrm{PAH}$, and may aid in identifying new therapeutic strategies. Additional insights into the altered pulmonary endothelial communication with both resident vascular cells (PA-SMCs, myofibroblasts, pericytes) and circulating cells (immune cells) are also a prerequisite for a better understanding of $\mathrm{PH} / \mathrm{PAH}$ pathogenesis.

The endothelium is also critical for the development of a functional vascular network that is dependent upon signals exchanged between the different cell types responsible for coordinating this process. In experimental and human $\mathrm{PH} / \mathrm{PAH}$, angiogenesis is disturbed with loss and progressive obliteration of pre-capillary arteries leading to a pattern of pulmonary vascular rarefaction ("dead-tree" picture), even if several pro-angiogenic factors are overabundant and/or overactive and an overexpression of Notch3 signalling in PA-SMCs has been reported. Therefore, further studies are needed to identify how the endothelial microenvironment, at the cell-cell and cell-matrix interfaces, impairs pulmonary endothelial integrity and its regenerative angiogenic capacity in $\mathrm{PH} / \mathrm{PAH}$. In this context, a better knowledge of the contribution of circulating cells and resident vascular progenitors should be determined [22-28]. Indeed, pericytes are important not only for vessel maturation and stabilisation, but also for the prevention of endothelial sprout formation and endothelial proliferation required for the production of a new functional vasculature. In experimental and human $\mathrm{PH} / \mathrm{PAH}$, the total number of pulmonary pericytes in distal pulmonary arteries increases substantially during disease progression [26] and defects in pericyte function have been demonstrated [28]. Isolated pericytes from PAH patients exhibit reduced levels of genes crucial for the Wnt/planar cell polarity pathway and fail to associate with endothelial cells during tube formation as assessed in Matrigel tube formation assays [28].

It is now clearly established that abnormal BMPR2 signalling can adversely impact endothelial barrier function, DNA lesion persistence related to impaired DNA repair [29], metabolism, mitochondrial fission and fusion [30, 31], and also inflammation and its resolution [32-34]. Therefore, much effort should be 
made to better understand the interplay between the BMPR2 signalling system and the process of pulmonary vascular remodelling.

\section{Accumulation of PA-SMCs and adventitial fibroblasts}

In $\mathrm{PAH}$, the pulmonary arterial microenvironment and the presence of several inherent intrinsic abnormalities and dysregulated signals are known to partly explain the progressive accumulation of resident PA-SMCs and adventitial fibroblasts. In recent years, pre-clinical and early-stage clinical efforts have highlighted emerging targets in PAH pathophysiology. As in endothelial cells, DNA damage response pathways are critically implicated in PA-SMC and fibroblast survival in PAH. In both human and experimental PH/PAH the decrease in BRCA1 (breast cancer 1) protein following BMPR2 downregulation is associated with the upregulation of poly(ADP ribose) polymerase 1 (PARP1) in response to the increase in DNA damage insults [35]. The upregulation of PARP1 in PAH PA-SMCs allows them to cope with the environmental stresses by damping DNA damage consequences, adapting their mitochondrial functions into a survival mode $[36,37]$. Inhibition of PARP1 in experimental PH models has shown greater efficacy than the combination of current standard of care, and thus the US Food and Drug Administration-approved PARP1 inhibitor olaparib is under clinical investigation in PAH (ClinicalTrials. gov identifier NCT03251872). Another advance derives from observations of a downregulation of miRNA-124 leading to an upregulation of the RNA splicing factor polypyrimidine tract binding protein 1 (PTBP1) in both fibroblasts and endothelial cells in the PH vasculature [38, 39]. PTBP1, along with other members of the heterogeneous nuclear ribonucleoprotein family of splicing factors, has been demonstrated to regulate splicing of pyruvate kinase muscle (PKM) isoforms. Increases in PTBP1 lead to increased accumulation of the PKM2 isoform, which in its dimeric (unactivated state) promotes glycolysis, proliferation and apoptosis resistance even in aerobic environments. Restoration of a normal PKM2/PKM1 ratio using pharmacological inhibitors attenuated fibroblast and endothelial cell proliferation in vitro and in vivo. In addition, it has been found that tumour necrosis factor- $\alpha$ (TNF- $\alpha$ ) inhibits BMPR2 expression and promotes post-translational cleavage via the "a disintegrin and metalloproteases" ADAM10 and ADAM17 in PA-SMCs, favouring BMP-mediated proliferation via alternative activin receptors [40]. Furthermore, exposure of PA-SMCs to high glucose increases expression of SMURF1, an E3 ubiquitin-protein ligase that dampens BMP signalling, and decreases phospho-Smad1/5/8, mimicking signalling patterns in mutation-negative PAH-derived PA-SMCs, which are normalised by blocking glucose uptake [41]. Similarly, Smad3 depletion in PA-SMCs and in pulmonary endothelial cells was found to contribute to the heightened proliferation and migration, which was attenuated by inhibition of myocardin-related transcriptional factor [42].

Other promising targets have also been recently identified in this context and include, among others: leukotriene B4 (LTB4), interleukin-6 (IL-6) and leptin receptors, as well as the transcriptional corepressor C-terminal binding protein 1 (CtBP1), transforming growth factor- $\beta$, peroxisome proliferator-activated receptor- $\gamma$ (PPAR- $\gamma$ ), mammalian target of rapamycin complex 1 (mTORC1) and Forkhead box O1 (FoxO1) pathways [43-47]. In PAH, it is also established that the dynamic and unadapted remodelling of the extracellular matrix forms a permissive milieu that not only favours cell motility, proliferation, apoptosis, and differentiation of resident vascular cells and recruitment of inflammatory cells, but can also have a considerable effect on vessel stiffness [48-51]. Owing to their close locations, and because there are several lines of evidence that indicate the existence of a complex interrelationship between pulmonary arterial cells and perivascular monocytes and macrophages [52, 53], a more complete understanding of these complex interrelationships is needed.

\section{Dysregulation of the innate and adaptive immune system}

In experimental $\mathrm{PH}$, perivascular inflammatory infiltrates of mixed inflammatory cells often precede the structural pulmonary vascular remodelling, supporting the notion that maladaptation of the inflammatory and immune systems exists and contributes to remodelling. Consistent with this notion, small lymphoid aggregates to large accumulations of lymphocytes resembling highly organised lymphoid follicles can be observed in lungs of patients with PAH. Similarly, it is now established that circulating levels of inflammatory mediators correlate with a worse clinical outcome in $\mathrm{PAH}$ and that alterations of circulating cell subsets can be observed [54-56]. As a result, therapies that directly modulate inflammatory processes have become a recent focus of clinical studies in PAH.

The fact that steroid or aspirin treatment is not effective in IPAH and HPAH, and that prostacyclin, which has anti-inflammatory properties [57], does not reverse the pulmonary vascular remodelling underscores the fact that additional insights into the roles played by the immune cells and key cytokines/chemokines are a prerequisite for developing novel therapeutic strategies. Recent investigations provide evidence that pulmonary vascular cells are important local sources of soluble signals in PAH that contribute to pulmonary vascular remodelling. Indeed, PA-SMCs, endothelial cells, fibroblasts and myofibroblasts from 
patients with PAH exhibit a marked pro-inflammatory signature characterised by heightened expression of various cytokines and chemokines, and of key inflammatory cell adhesion molecules, such as ICAM1. The excessive local secretion of IL-1, IL-6, LTB4, macrophage migration inhibitory factor, leptin and TNF- $\alpha$, and the inactivation of FoxO1, play an integral role in mediating the structural and functional changes in the pulmonary vasculature in PAH $[12,40,43-46,58]$.

Impaired T-regulatory cell function, T-helper 17 cell immune polarisation [59] and dendritic cell recruitment in pulmonary vascular lesions have been demonstrated in tissues from PAH patients, supporting maladaptation of the immune response. Accordingly, circulating autoantibodies are commonly detected in PAH patients without evidence of an associated autoimmune condition. Furthermore, lymphoid neogenesis in PH/PAH lungs has been reported. Moving forward, a better understanding of the mechanisms leading to alteration in the balance between immunity and tolerance in PAH may allow the identification of immunopathological approaches to PAH management.

Additional contributing factors to the dysregulation of the innate and adaptive immune system in PAH include, among others: shear stress, chronic exposure to hypoxia, dysregulation in BMPR2 signalling, ageing of the pulmonary circulation, metabolic derangements, dysfunctional or distressed mitochondria, circulating autoantibodies and immune complexes. Indeed, environmental or genotoxic stresses favour pulmonary vascular remodelling through the activation of vascular, inflammatory and immune cells. Recently, perivascular immune complexes containing the antiviral protein SAMHD1 (SAM domain and HD domain-containing protein 1) has been found to be an innate immune response to an unexplained elevation in sequences and protein products of the human endogenous retrovirus $\mathrm{K}$ observed in $\mathrm{PAH}$ perivascular macrophages and circulating monocytes [60]. These abnormalities, in addition to others, may perpetuate a dysregulated immune and inflammatory response (figure 5). Unadapted immunity and inflammation appear to play an active role in pulmonary endothelial dysfunction and vascular remodelling in $\mathrm{PH} / \mathrm{PAH}$, and are also likely to have a detrimental effect on cardiac function. However, there are subtleties and complexities that require further investigation to determine whether and which anti-inflammatory strategies will be best suited to treat $\mathrm{PAH}$.

The prominent roles of autoimmunity and inflammation in the pathogenesis of PAH warrant future clinical studies of newer biological agents that can target specific inflammatory pathways. Several clinical trials are currently exploring the efficacy and safety of different anti-inflammatory agents in PAH; these include, among others: rituximab, a chimeric anti-human CD20 (ClinicalTrials.gov identifier NCT01086540) in patients with systemic sclerosis-associated PAH; tocilizumab, a humanised anti-IL-6

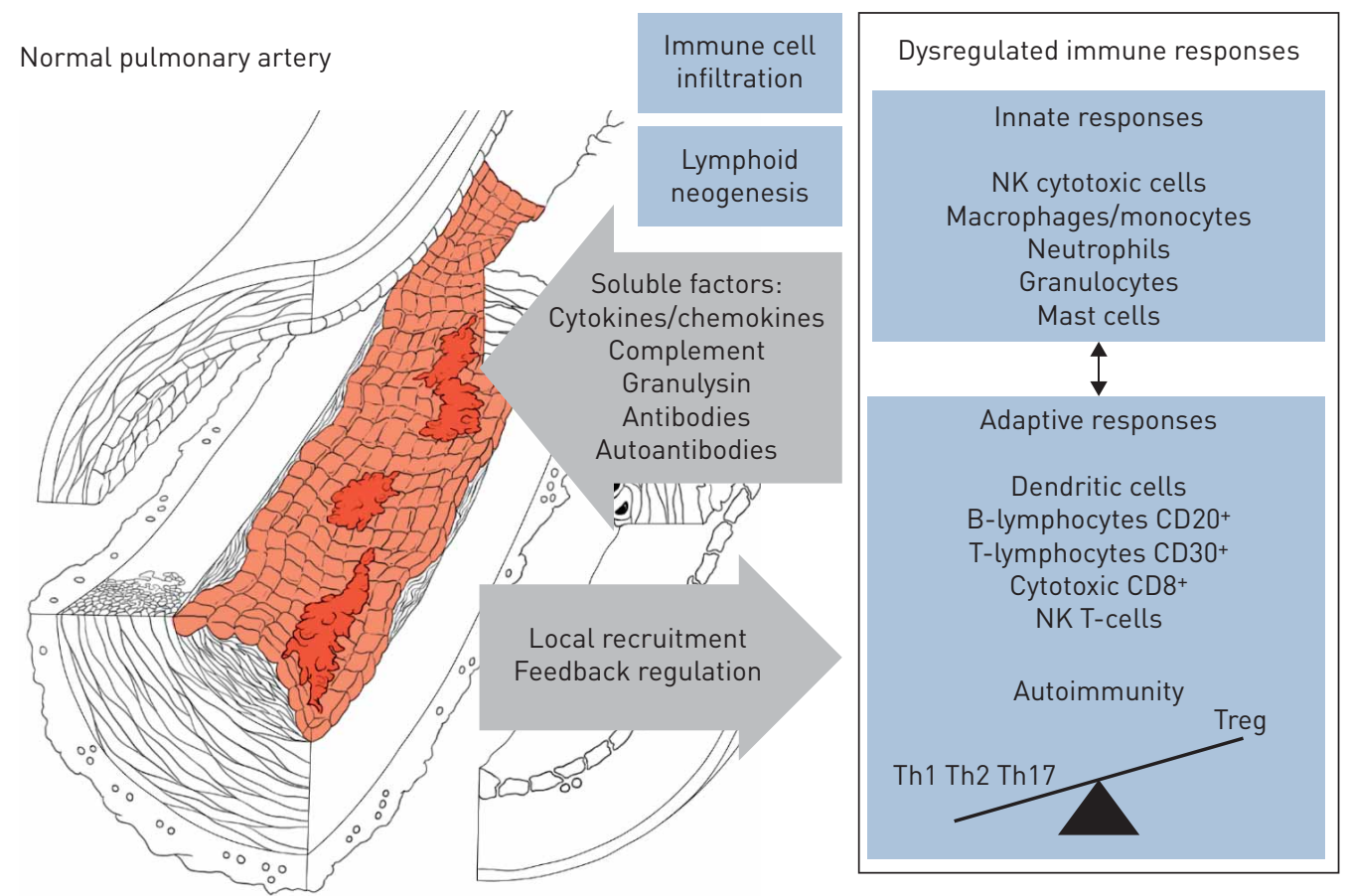

PAH pulmonary artery

FIGURE 5 Schematic representation of immune disturbance associated with pulmonary arterial hypertension (PAH) and pulmonary vascular remodelling. NK: natural killer; Th: T-helper; Treg: T-regulatory. 
receptor antibody (ClinicalTrials.gov identifier NCT02676947) in PAH patients; FK506, an inhibitor of calcineurin and a binding partner of FKBP12 (12-kDa FK506-binding protein) that has also been shown to upregulate BMPR2 expression; and the elastase inhibitor produced as a recombinant protein.

\section{Recent advances in molecular mechanisms and emerging therapeutic targets}

Although much remains to be understood, decades of extensive studies have related PAH pathobiology to genetic, epigenetic and environmental factors (viruses, drugs, toxins, hypoxia and inflammation) that can cause or accelerate irreversible remodelling of the pulmonary vascular bed. We propose that the genetic, epigenetic and environmental factors lead to deregulation of growth factors, ion channels, hormones and cytokines that subsequently activate a complex cascade of signalling pathways causing abnormalities in vascular cell phenotype, including proliferation, differentiation/de-differentiation and inflammation. This suggests that transcriptional dysregulation in the pulmonary vasculature can be an early event that shapes the pulmonary vascular transcriptome, causing both depletion and ectopic activation of gene products that eventually lead to aberrant cellular processes and consequently adverse vascular remodelling [61]. How cells sense and respond to environmental triggers that lead to transcriptional dysregulation remains a central question of pulmonary vascular research. Recent studies are providing new insights that include: the role of non-receptor kinases; the potential of ion channels to control pulmonary arterial tone and vascular remodelling processes; the disruption in the activity of gene expression regulators (i.e. transcription factors and transcriptional coregulators); the epigenetic processes resulting in aberrant activation of chromatin-remodelling proteins, non-coding and microRNAs; and the severe metabolic perturbations that affect transcription, post-transcriptional processes and signalling pathways (figure 6).

\section{Dysregulation of receptor and non-receptor kinase signalling}

In $\mathrm{PH} / \mathrm{PAH}$, altered expression and function of different growth factors and their respective receptor tyrosine kinases (e.g. fibroblast growth factor 2, vascular endothelial growth factor, platelet-derived growth factor, epidermal growth factor and nerve growth factor) together with inflammatory mediators (e.g. cytokines, chemokines, circulating autoantibodies and immune complexes) contribute to the phenotypic alterations of resident pulmonary vascular cells, and their accumulation in the wall of distal pulmonary arteries has been implicated.

\section{Emerging ion channel targets}

The identification of heterozygous loss-of-function mutations in the KCNK3 (potassium channel subfamily K member 3) gene that encodes TWIK-related acid-sensitive potassium channel 1 (TASK1) as a

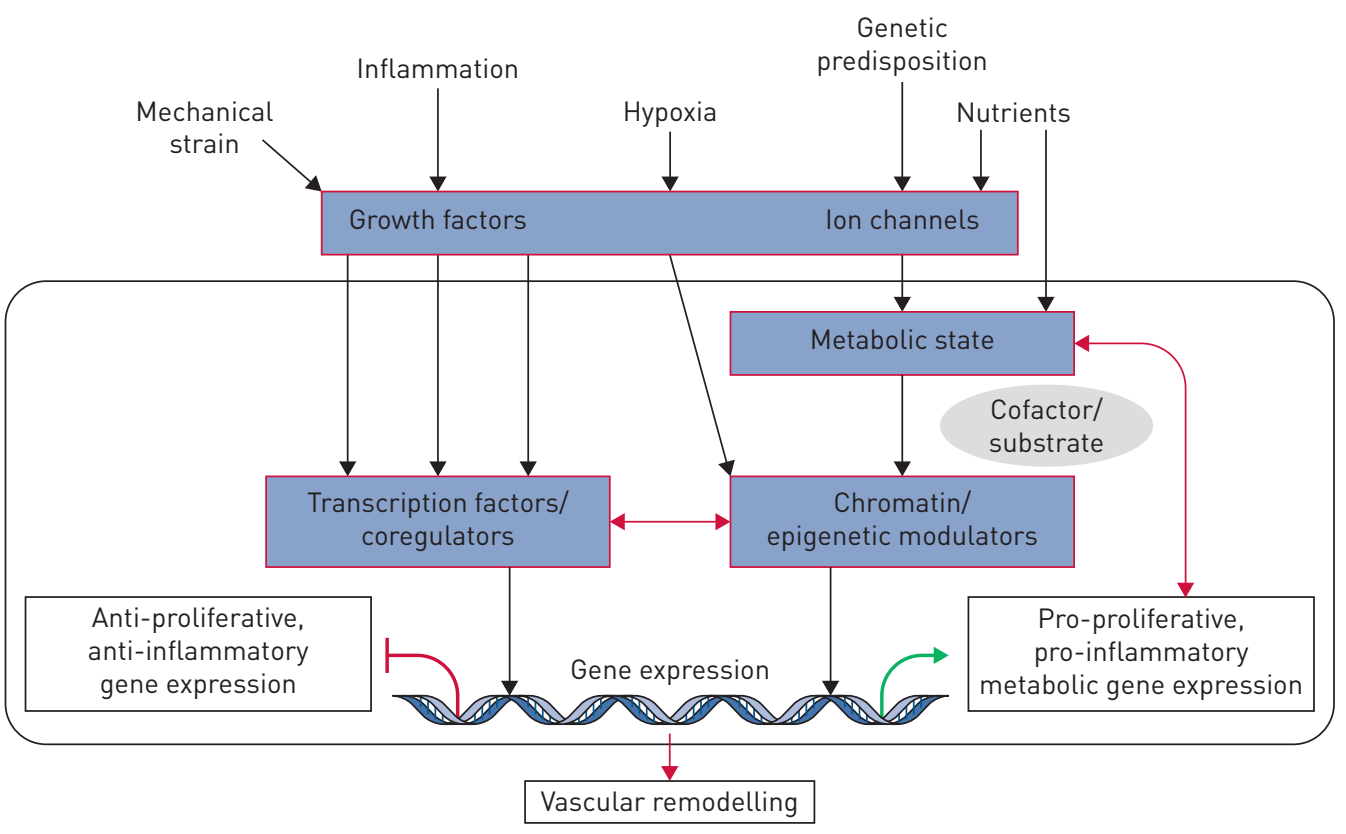

FIGURE 6 Cross-talk between transcription factors, epigenetics and metabolism in pulmonary hypertension. Growth factors, ion channels, hormones and cytokines activate the classical signalling pathways and downstream transcriptional factors, which will recruit chromatin-modifying enzymes to local chromatin. On the other hand, nutrient levels and cell metabolism will affect levels of the metabolites, which are required substrates of chromatin-modifying enzymes that use these metabolites to modify both histones and DNA. Variations in these inputs will determine epigenome remodelling and transcription, and subsequently vascular remodelling. 
cause for PAH has revived interest in the concept of channelopathy [62]. In addition to voltage-gated potassium channels, different types of transient receptor potential channels, calcium sensor proteins and calcium-activated chloride channels have been implicated in PAH pathogenesis [63-67]. The dysregulation of potassium channels could have a central role in the immediate and long-term regulation of pulmonary vascular function in $\mathrm{PAH}[68,69]$. This notion is consistent with the fact that restoration of potassium channel function can prevent or reverse experimental $\mathrm{PH}$. For example, in vivo pharmacological activation of KCNK3 has beneficial effects in monocrotaline-induced $\mathrm{PH}$ [69]. Interestingly, endothelin, serotonin (5-HT), oxidative stress, BMPR2, docosahexaenoic acid and growth factors such as platelet-derived growth factor are known modulators of potassium channel activities [70]. Furthermore, inhibition of voltage-gated potassium channels could represent one potential mechanism involved in some drug-induced PH [19, 71]. The current challenge is to identify small molecules or specific strategies to restore the expression and/or activity of these ion channels in PAH dysfunctional pulmonary vasculature.

\section{Key transcription factors and transcriptional coregulators}

Numerous transcription factors and transcriptional coactivators (defined as a type of protein that itself has no DNA-binding activity, but can bind to a transcription factor to augment or repress the transcription factor's ability to activate gene expression) have been implicated in $\mathrm{PH}$ and right ventricular dysfunction. Some of the transcription factors include PPAR- $\gamma$, myocyte enhancer factor 2 (MEF2), FoxO, p53, KLF4, HIFs, CCAAT-enhancer binding proteins (CEBPs), Runt-related transcription factor 2 (RUNX2), activator protein 1 (AP-1), CtBP1, FoxM1, PKM2, NF- $\kappa B, \beta$-catenin, the Twist family basic helix-loop-helix transcription factor 1 (TWIST1) and SLUG (figure 7) [45, 51, 72-77]. FoxO1 isoform inactivation is involved in the pro-proliferative and apoptosis-resistant phenotype of PA-SMCs, and is a known downstream mediator of different growth factors and inflammatory mediators [45]. Furthermore, the related transcription factor FoxM1 promotes PA-SMC accumulation in $\mathrm{PH}$ [77], suggesting that targeting the FoxO-FoxM1 axis could be a viable strategy for treatment of $\mathrm{PH}$. Transcription factor coactivators have also been recently implicated in PH pathophysiology. These include PKM2 and CtBP1 [39, 72]. Importantly, normalising metabolic activity via metabolic inhibitors such as 2-deoxyglucose or directly reducing CtBP1 expression attenuates $\mathrm{PH}$ fibroblast proliferation and apoptosis resistance [72].

Abnormalities in other transcription factors, such as Notch3, signal transducer and activator of transcription 3 (STAT3), and the HIPPO central component large tumour suppressor 1 (LAST1) [78], and in various growth factors, underlie phenotypic changes in pulmonary vascular cells in PAH. For example, loss of PPAR- $\gamma$ in pulmonary endothelial cells leads to a deficient complex with $\beta$-catenin and this results in reduced apelin, causing impaired pulmonary endothelial cell survival and angiogenesis [79]. Similarly, Yes-associated protein (YAP)/transcriptional coactivator with PDZ-binding motif (TAZ) are also emerging as key regulators of cell growth and migration in $\mathrm{PAH}$ and link mechanical stimuli to dysregulated vascular metabolism [80]. A comparison of two rat strains, F344 and WKY, which differ in their response to chronic hypoxia, highlighted the gene Slc39a12 that encodes the zinc transporter ZIP12 as another major regulator of hypoxia-induced pulmonary vascular remodelling [81]. Although a more complete understanding of the overall risk-benefit ratio of these different strategies needs to be evaluated, these data reveal the potential therapeutic interest for targeting particular transcription factors and/or transcription factor coactivators in $\mathrm{PH} / \mathrm{PAH}$.

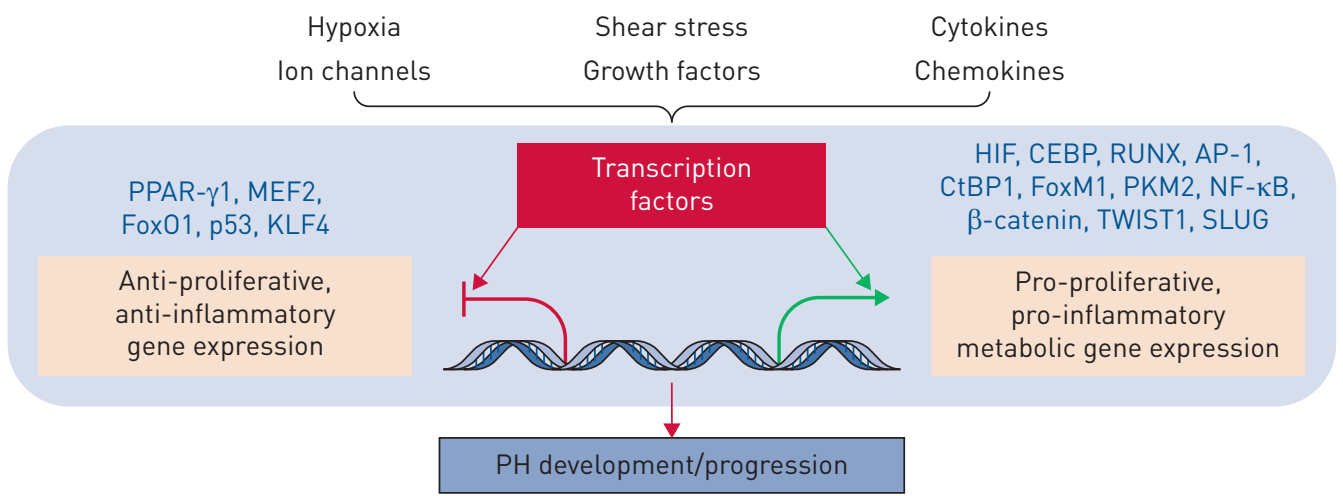

FIGURE 7 Role of transcription factors and transcriptional coregulators in the pathogenesis of pulmonary hypertension (PH). See main text for definitions. Multiple pathological stimuli, such as hypoxia, shear stress, oxidative stress, mitogens and inflammation (cytokines and chemokines), trigger downstream signalling cascades, which modulate the recruitment and activation of transcription factors and transcriptional coregulators that determine the stimulus-specific transcriptional responses in $\mathrm{PH}$. 
Emerging roles for epigenetic dysregulation

Altered DNA methylation of superoxide dismutase 2 and granulysin genes, histone H1 levels, aberrant expression levels of histone deacetylases (HDACs) and bromodomain-containing protein 4, and dysregulated microRNA and long-non-coding RNA networks together suggest multiple levels of epigenetic involvement in $\mathrm{PAH}$ pathogenesis. Recently, clinical interest in HDAC inhibition in PAH has been regenerated through the discovery that the cytosolic HDAC6 is implicated in both pulmonary arterial remodelling and right ventricular failure. Its inhibition using a HDAC6-specific inhibitor could be tested [82]. Therefore, a complete understanding of the mechanisms involved in altered gene expression in diseased cells [61] is vital for the design of novel therapeutic strategies. Mice lacking sirtuin 3 (SIRT3), a mitochondrial deacetylase, develop spontaneous PH. Interestingly, these mice have increased acetylation and inhibition of many mitochondrial enzymes and complexes, suppressing mitochondrial function. Moreover, a loss-of-function SIRT3 polymorphism is associated with PAH [83]. These studies suggest that mitochondrial products such as 2 -hydroxyglutarate, $\alpha$-ketoglutarate, citrate or acetyl-CoA may regulate transcription factors and epigenetic mechanisms. This metabolism-epigenetics axis facilitates adaptation to a changing environment in the pulmonary vasculature and right ventricle, providing a potential novel therapeutic target. In summary, an integrative understanding of the interplay between the molecular, metabolic, genetic and epigenetic rewiring in $\mathrm{PH} / \mathrm{PAH}$ is far from complete, but conceptual themes are beginning to emerge.

\section{Metabolic remodelling and mitochondrial dysfunction}

Aberrant signalling in pulmonary vascular cells that contributes to $\mathrm{PH} / \mathrm{PAH}$ development and progression may be the cause or consequence of metabolic dysregulation. Several metabolic and signalling pathways could be interesting targets for PH/PAH therapy, and those being investigated involve modulation of HIF1 and the phosphoinositide 3-kinase/protein kinase $\mathrm{B} / \mathrm{mammalian}$ target of rapamycin pathway, mitochondrial phosphatase and tensin homolog (PTEN)-induced kinase 1 (PINK1), the HIPPO and p53 signalling pathways, and inhibition of pyruvate dehydrogenase kinase (PDK), an inhibitor of the mitochondrial enzyme pyruvate dehydrogenase ( $\mathrm{PDH}$, the gatekeeping enzyme of glucose oxidation). Restitution of oxidative metabolism with the use of dichloroacetate in PAH patients might be efficacious in a subset of patients [84]. Lack of clinical response was associated with the presence of functional variants of SIRT3 and UCP2 (uncoupling protein 2) that predict reduced PDH function independent of PDK and greater resistance to dichloroacetate [84]. Recently, accumulation of mitochondrial heat shock protein 90 has been found to contribute to the heightened aerobic glycolysis and to the mitochondrial stress response in cultured PA-SMCs from PAH patients [85]. Even if further investigations are required to better understand the contribution of different inflammatory processes, high shear stress, chronic hypoxia and certain hormones in these metabolic dysregulations, and to identify additional and novel mechanistic targets, the use of metabolism-based therapies may be promising for PH/PAH.

\section{Current and future perspectives}

Animal model systems that mimic specific processes contributing to $\mathrm{PH}$ could be valuable to discover new treatment targets and to study their contribution to the disease pathogenesis at early and late phases. However, it is also clear that pulmonary vascular lesions experimentally induced do not recapitulate the full spectrum of the human disease, and there are interspecies, age, sex and environment differences in the responses to stimuli used to promote $\mathrm{PH}$ in these different models. In addition, there are differences in the anatomical and functional development of the immune system in animals compared with humans. The use of human tissue samples or iPSCs to derive endothelial cells or SMCs from PAH patients should thus complement animal studies in providing a path to move $\mathrm{PAH}$ treatment into the clinic. While there are clearly limits to model $\mathrm{PH}$, there are several measures that should improve predictive utility and translational efficiency: 1) define the research problem as precisely as possible; 2) identify the strengths and weaknesses of each currently available PH model; and 3) identify how they may best be used. Improvements can be made in randomising animals, consideration of both sexes, and blinding observations related to haemodynamic and structural end-points [86-88].

Considerable advances are possible by interrogating large datasets to find novel pathways, critical transcription factors, microRNAs, biomarkers and metabolic mediators of $\mathrm{PAH}$ as well as points of intersection that can help develop better targeted therapies. Bioinformatic approaches with established datasets can generate a PAH signature and an anti-signature that can be used to find novel or repurposed therapies, as well as to deliver them. In addition to consideration of shear stress levels, the nature of the cell matrix and the nature of cell interactions need to be incorporated. For this reason, engineering systems that use all three layers of the vessel wall and that mimic the extracellular matrix produced by these cells will be most informative. New opportunities such as three-dimensional printing can now produce extensive vascular networks. 
Translating exciting pre-clinical discoveries into clinical testing presents special challenges for a rare disease like PAH. First, because of the limited number of subjects, new approaches, gleaned from the laboratory, must be used as adjuvants on top of standard-of-care therapy. An additional challenge for researchers is to find common pathways or pathophysiological processes to safely target all forms of $\mathrm{PH} /$ PAH. In this line of investigation, analyses of data from publicly available databases of "PAH-omics" data (most commonly transcriptomes) and from pooled PAH registries could represent promising approaches to discover common modules of genes that reveal new therapies. Another appealing approach could be to move away from common pathways to, instead, phenotype patients into "responder" and "non-responder" groups. Here, the challenge is discovering patient groups, within a group that is already small to start with, who are particularly well suited to adjuvant therapy.

Conflict of interest: M. Humbert reports personal fees from Actelion and Merck, and grants and personal fees from Bayer and GSK, outside the submitted work. C. Guignabert has nothing to disclose. S. Bonnet has nothing to disclose. P. Dorfmüller reports personal fees from MSD, Bayer, Actelion and Roche, outside the submitted work. J.R. Klinger reports grants for animal study of extra cellular vesicles from United Therapeutics, outside the submitted work. M.R. Nicolls has nothing to disclose. A.J. Olschewski has nothing to disclose. S.S. Pullamsetti has nothing to disclose. R.T. Schermuly has nothing to disclose. K.R. Stenmark reports grants from ContraFect, personal fees for advisory board work from Pfizer, New York, personal fees for membership of a steering Committee Member (Entelligence Awards Program) from Actelion, and personal fees for scientific advisory board work from Janssen Research and Development, outside the submitted work. M. Rabinovitch reports use of FK506 for the treatment of PAH. The Board of Trustees of Leland Stanford Junior University, assignee. Patent PCT/US2012035793.

\section{References}

1 Fayyaz AU, Edwards WD, Maleszewski JJ, et al. Global pulmonary vascular remodeling in pulmonary hypertension associated with heart failure and preserved or reduced ejection fraction. Circulation 2017; 137: $1796-1810$.

2 Dorfmuller P, Gunther S, Ghigna MR, et al. Microvascular disease in chronic thromboembolic pulmonary hypertension: a role for pulmonary veins and systemic vasculature. Eur Respir J 2014; 44: 1275-1288.

3 Galambos C, Sims-Lucas S, Abman SH, et al. Intrapulmonary bronchopulmonary anastomoses and plexiform lesions in idiopathic pulmonary arterial hypertension. Am J Respir Crit Care Med 2016; 193: 574-576.

4 Ghigna MR, Guignabert C, Montani D, et al. BMPR2 mutation status influences bronchial vascular changes in pulmonary arterial hypertension. Eur Respir J 2016; 48: 1668-1681.

5 Montani D, Lau EM, Dorfmuller P, et al. Pulmonary veno-occlusive disease. Eur Respir J 2016; 47: 1518-1534.

6 Galiè N, Kim NH. Pulmonary microvascular disease in chronic thromboembolic pulmonary hypertension. Proc Am Thorac Soc 2006; 3: 571-576.

7 Nossent EJ, Antigny F, Montani D, et al. Pulmonary vascular remodeling patterns and expression of general control nonderepressible 2 (GCN2) in pulmonary veno-occlusive disease. J Heart Lung Transplant 2018; 37: 647-655.

8 Huertas A, Guignabert C, Barbera JA, et al. Pulmonary vascular endothelium: the orchestra conductor in respiratory diseases: highlights from basic research to therapy. Eur Respir J 2018; 51: 1700745.

9 Hopper RK, Moonen JR, Diebold I, et al. In pulmonary arterial hypertension, reduced BMPR2 promotes endothelial-to-mesenchymal transition via HMGA1 and its target Slug. Circulation 2016; 133: 1783-1794.

10 Ranchoux B, Antigny F, Rucker-Martin C, et al. Endothelial-to-mesenchymal transition in pulmonary hypertension. Circulation 2015; 131: 1006-1018.

11 Stenmark KR, Frid M, Perros F. Endothelial-to-mesenchymal transition: an evolving paradigm and a promising therapeutic target in PAH. Circulation 2016; 133: 1734-1737.

12 Le Hiress M, Tu L, Ricard N, et al. Proinflammatory signature of the dysfunctional endothelium in pulmonary hypertension: role of the macrophage migration inhibitory factor/CD74 complex. Am J Respir Crit Care Med 2015; 192: 983-997.

13 Gu M, Shao NY, Sa S, et al. Patient-specific iPSC-derived endothelial cells uncover pathways that protect against pulmonary hypertension in BMPR2 mutation carriers. Cell Stem Cell 2017; 20: 490-504.

14 Sa S, Gu M, Chappell J, et al. iPSC model of pulmonary arterial hypertension reveals novel gene expression and patient specificity. Am J Respir Crit Care Med 2017; 195: 930-941.

15 Abe K, Shinoda M, Tanaka M, et al. Haemodynamic unloading reverses occlusive vascular lesions in severe pulmonary hypertension. Cardiovasc Res 2016; 111: 16-25.

16 Szulcek R, Happe CM, Rol N, et al. Delayed microvascular shear adaptation in pulmonary arterial hypertension. role of platelet endothelial cell adhesion molecule-1 cleavage. Am J Respir Crit Care Med 2016; 193: 1410-1420.

17 Dai Z, Li M, Wharton J, et al. Prolyl-4 hydroxylase 2 (PHD2) deficiency in endothelial cells and hematopoietic cells induces obliterative vascular remodeling and severe pulmonary arterial hypertension in mice and humans through hypoxia-inducible factor-2alpha. Circulation 2016; 133: 2447-2458.

18 Chen PI, Cao A, Miyagawa K, et al. Amphetamines promote mitochondrial dysfunction and DNA damage in pulmonary hypertension. JCI Insight 2017; 2: e90427.

19 Guignabert C, Phan C, Seferian A, et al. Dasatinib induces lung vascular toxicity and predisposes to pulmonary hypertension. J Clin Invest 2016; 126: 3207-3218.

20 Daccord C, Letovanec I, Yerly P, et al. First histopathological evidence of irreversible pulmonary vascular disease in dasatinib-induced pulmonary arterial hypertension. Eur Respir J 2018; 51: 1701694.

21 Montani D, Bergot E, Gunther S, et al. Pulmonary arterial hypertension in patients treated by dasatinib. Circulation 2012; 125: 2128-2137.

22 Aliotta JM, Pereira M, Amaral A, et al. Induction of pulmonary hypertensive changes by extracellular vesicles from monocrotaline-treated mice. Cardiovasc Res 2013; 100: 354-362. 
23 Aliotta JM, Pereira M, Wen S, et al. Exosomes induce and reverse monocrotaline-induced pulmonary hypertension in mice. Cardiovasc Res 2016; 110: 319-330.

24 de Mendonca L, Felix NS, Blanco NG, et al. Mesenchymal stromal cell therapy reduces lung inflammation and vascular remodeling and improves hemodynamics in experimental pulmonary arterial hypertension. Stem Cell Res Ther 2017; 8: 220 .

25 Dierick F, Hery T, Hoareau-Coudert B, et al. Resident $\mathrm{PW} 1^{+}$progenitor cells participate in vascular remodeling during pulmonary arterial hypertension. Circ Res 2016; 118: 822-833.

26 Ricard N, Tu L, Le Hiress M, et al. Increased pericyte coverage mediated by endothelial-derived fibroblast growth factor-2 and interleukin-6 is a source of smooth muscle-like cells in pulmonary hypertension. Circulation 2014; 129: $1586-1597$.

27 Yan L, Chen X, Talati M, et al. Bone marrow-derived cells contribute to the pathogenesis of pulmonary arterial hypertension. Am J Respir Crit Care Med 2016; 193: 898-909.

28 Yuan K, Orcholski ME, Panaroni C, et al. Activation of the Wnt/planar cell polarity pathway is required for pericyte recruitment during pulmonary angiogenesis. Am J Pathol 2015; 185: 69-84.

29 Li M, Vattulainen S, Aho J, et al. Loss of bone morphogenetic protein receptor 2 is associated with abnormal DNA repair in pulmonary arterial hypertension. Am J Respir Cell Mol Biol 2014; 50: 1118-1128.

30 Chen NY, Collum SD, Luo F, et al. Macrophage bone morphogenic protein receptor 2 (BMPR2) depletion in idiopathic pulmonary fibrosis (IPF) and group III pulmonary hypertension. Am J Physiol Lung Cell Mol Physiol 2016: 311: L238-L254.

31 Diebold I, Hennigs JK, Miyagawa K, et al. BMPR2 preserves mitochondrial function and DNA during reoxygenation to promote endothelial cell survival and reverse pulmonary hypertension. Cell Metab 2015; 21: 596-608.

32 Sawada H, Saito T, Nickel NP, et al. Reduced BMPR2 expression induces GM-CSF translation and macrophage recruitment in humans and mice to exacerbate pulmonary hypertension. J Exp Med 2014; 211: 263-280.

33 Soon E, Crosby A, Southwood M, et al. Bone morphogenetic protein receptor type II deficiency and increased inflammatory cytokine production. A gateway to pulmonary arterial hypertension. Am J Respir Crit Care Med 2015; 192: 859-872.

34 Hwangbo C, Lee HW, Kang H, et al. Modulation of endothelial BMPR2 activity by VEGFR3 in pulmonary arterial hypertension. Circulation 2017; 135: 2288-2298.

35 Ranchoux B, Meloche J, Paulin R, et al. DNA damage and pulmonary hypertension. Int J Mol Sci 2016; 17: E990.

36 Meloche J, Le Guen M, Potus F, et al. miR-223 reverses experimental pulmonary arterial hypertension. Am J Physiol Cell Physiol 2015; 309: C363-C372.

37 Meloche J, Pflieger A, Vaillancourt M, et al. Role for DNA damage signaling in pulmonary arterial hypertension. Circulation 2014; 129: 786-797.

38 Caruso P, Dunmore BJ, Schlosser K, et al. Identification of microRNA-124 as a major regulator of enhanced endothelial cell glycolysis in pulmonary arterial hypertension via PTBP1 (polypyrimidine tract binding protein) and pyruvate kinase M2. Circulation 2017; 136: 2451-2467.

39 Zhang H, Wang D, Li M, et al. Metabolic and proliferative state of vascular adventitial fibroblasts in pulmonary hypertension is regulated through a microRNA-124/PTBP1 (polypyrimidine tract binding protein 1)/pyruvate kinase muscle axis. Circulation 2017; 136: 2468-2485.

40 Hurst LA, Dunmore BJ, Long L, et al. TNFalpha drives pulmonary arterial hypertension by suppressing the BMP type-II receptor and altering NOTCH signalling. Nat Commun 2017; 8: 14079

41 Barnes JW, Kucera ET, Tian L, et al. Bone morphogenic protein type 2 receptor mutation-independent mechanisms of disrupted bone morphogenetic protein signaling in idiopathic pulmonary arterial hypertension. Am J Respir Cell Mol Biol 2016; 55: 564-575.

42 Zabini D, Granton E, Hu Y, et al. Loss of SMAD3 promotes vascular remodeling in pulmonary arterial hypertension via MRTF disinhibition. Am J Respir Crit Care Med 2017; 196: 1411-1421.

43 Huertas A, Tu L, Thuillet R, et al. Leptin signalling system as a target for pulmonary arterial hypertension therapy. Eur Respir J 2015; 45: 1066-1080.

44 Qian J, Tian W, Jiang X, et al. Leukotriene B4 activates pulmonary artery adventitial fibroblasts in pulmonary hypertension. Hypertension 2015; 66: 1227-1239.

45 Savai R, Al-Tamari HM, Sedding D, et al. Pro-proliferative and inflammatory signaling converge on FoxO1 transcription factor in pulmonary hypertension. Nat Med 2014; 20: 1289-1300.

46 Tamura Y, Phan C, Tu L, et al. Ectopic upregulation of membrane-bound IL6R drives vascular remodeling in pulmonary arterial hypertension. J Clin Invest 2018; 128: 1956-1970.

47 Yung LM, Nikolic I, Paskin-Flerlage SD, et al. A selective transforming growth factor-beta ligand trap attenuates pulmonary hypertension. Am J Respir Crit Care Med 2016; 194: 1140-1151.

48 Jia D, He Y, Zhu Q, et al. RAGE-mediated extracellular matrix proteins accumulation exacerbates HySu-induced pulmonary hypertension. Cardiovasc Res 2017; 113: 586-597.

49 Chang YT, Chan CK, Eriksson I, et al. Versican accumulates in vascular lesions in pulmonary arterial hypertension. Pulm Circ 2016; 6: 347-359.

50 Tojais NF, Cao A, Lai YJ, et al. Codependence of bone morphogenetic protein receptor 2 and transforming growth factor-beta in elastic fiber assembly and its perturbation in pulmonary arterial hypertension. Arterioscler Thromb Vasc Biol 2017; 37: 1559-1569.

51 Ruffenach G, Chabot S, Tanguay VF, et al. Role for Runt-related transcription factor 2 in proliferative and calcified vascular lesions in pulmonary arterial hypertension. Am J Respir Crit Care Med 2016; 194: 1273-1285.

52 Hashimoto-Kataoka T, Hosen N, Sonobe T, et al. Interleukin-6/interleukin-21 signaling axis is critical in the pathogenesis of pulmonary arterial hypertension. Proc Natl Acad Sci USA 2015; 112: E2677-E2686.

53 Pugliese SC, Kumar S, Janssen WJ, et al. A time- and compartment-specific activation of lung macrophages in hypoxic pulmonary hypertension. J Immunol 2017; 198: 4802-4812.

54 Aldabbous L, Abdul-Salam V, McKinnon T, et al. Neutrophil extracellular traps promote angiogenesis: evidence from vascular pathology in pulmonary hypertension. Arterioscler Thromb Vasc Biol 2016; 36: $2078-2087$.

55 Harbaum L, Baaske KM, Simon M, et al. Exploratory analysis of the neutrophil to lymphocyte ratio in patients with pulmonary arterial hypertension. BMC Pulm Med 2017; 17: 72. 
56 Ozpelit E, Akdeniz B, Ozpelit ME, et al. Prognostic value of neutrophil-to-lymphocyte ratio in pulmonary arterial hypertension. J Int Med Res 2015; 43: 661-671.

57 Wang JW, Vu C, Poloso NJ. A prostacyclin analog, cicaprost, exhibits potent anti-inflammatory activity in human primary immune cells and a uveitis model. J Ocul Pharmacol Ther 2017; 33: 186-192.

58 Parpaleix A, Amsellem V, Houssaini A, et al. Role of interleukin-1 receptor 1/MyD88 signalling in the development and progression of pulmonary hypertension. Eur Respir J 2016; 48: 470-483.

59 Hautefort A, Girerd B, Montani D, et al. T-helper 17 cell polarization in pulmonary arterial hypertension. Chest 2015; 147: 1610-1620.

60 Saito T, Miyagawa K, Chen SY, et al. Upregulation of human endogenous retrovirus-K is linked to immunity and inflammation in pulmonary arterial hypertension. Circulation 2017; 136: 1920-1935.

61 Rhodes CJ, Im H, Cao A, et al. RNA sequencing analysis detection of a novel pathway of endothelial dysfunction in pulmonary arterial hypertension. Am J Respir Crit Care Med 2015; 192: 356-366.

62 Ma L, Roman-Campos D, Austin ED, et al. A novel channelopathy in pulmonary arterial hypertension. $N$ Engl J Med 2013; 369: 351-361.

63 Song S, Ayon RJ, Yamamura A, et al. Capsaicin-induced $\mathrm{Ca}^{2+}$ signaling is enhanced via upregulated TRPV1 channels in pulmonary artery smooth muscle cells from patients with idiopathic PAH. Am J Physiol Lung Cell Mol Physiol 2017; 312: L309-L325.

64 Fernandez RA, Wan J, Song S, et al. Upregulated expression of STIM2, TRPC6, and Orai2 contributes to the transition of pulmonary arterial smooth muscle cells from a contractile to proliferative phenotype. Am J Physiol Cell Physiol 2015; 308: C581-C593.

65 Song S, Carr SG, McDermott KM, et al. STIM2 (stromal interaction molecule 2)-mediated increase in resting cytosolic free $\mathrm{Ca}^{2+}$ concentration stimulates PASMC proliferation in pulmonary arterial hypertension. Hypertension 2018; 71: 518-529.

66 Hong Z, Chen KH, DasGupta A, et al. MicroRNA-138 and microRNA-25 down-regulate mitochondrial calcium uniporter, causing the pulmonary arterial hypertension cancer phenotype. Am J Respir Crit Care Med 2017; 195: 515-529.

67 Allawzi AM, Vang A, Clements RT, et al. Activation of anoctamin-1 limits pulmonary endothelial cell proliferation via p38-MAPK-dependent apoptosis. Am J Respir Cell Mol Biol 2018; 58: 658-667.

68 Lambert M, Boet A, Rucker-Martin C, et al. Loss of KCNK3 is a hallmark of RV hypertrophy/dysfunction associated with pulmonary hypertension. Cardiovasc Res 2018; 114: 880-893.

69 Antigny F, Hautefort A, Meloche J, et al. Potassium channel subfamily K member 3 (KCNK3) contributes to the development of pulmonary arterial hypertension. Circulation 2016; 133: 1371-1385.

70 Nagaraj C, Tang B, Nagy BM, et al. Docosahexaenoic acid causes rapid pulmonary arterial relaxation via KCa channel-mediated hyperpolarisation in pulmonary hypertension. Eur Respir J 2016; 48: 1127-1136.

71 Nagaraj C, Tang B, Balint Z, et al. Src tyrosine kinase is crucial for potassium channel function in human pulmonary arteries. Eur Respir J 2013; 41: 85-95.

72 Li M, Riddle S, Zhang $\mathrm{H}$, et al. Metabolic reprogramming regulates the proliferative and inflammatory phenotype of adventitial fibroblasts in pulmonary hypertension through the transcriptional corepressor C-terminal binding protein-1. Circulation 2016; 134: 1105-1121.

73 Calvier L, Chouvarine P, Legchenko E, et al. PPARgamma links BMP2 and TGFbetal pathways in vascular smooth muscle cells, regulating cell proliferation and glucose metabolism. Cell Metab 2017; 25: 1118-1134.

74 Kapitsinou PP, Rajendran G, Astleford L, et al. The endothelial prolyl-4-hydroxylase domain 2/hypoxia-inducible factor 2 axis regulates pulmonary artery pressure in mice. Mol Cell Biol 2016; 36: 1584-1594.

75 Ball MK, Waypa GB, Mungai PT, et al. Regulation of hypoxia-induced pulmonary hypertension by vascular smooth muscle hypoxia-inducible factor-1alpha. Am J Respir Crit Care Med 2014; 189: 314-324.

76 Pullamsetti SS, Kojonazarov B, Storn S, et al. Lung cancer-associated pulmonary hypertension: role of microenvironmental inflammation based on tumor cell-immune cell cross-talk. Sci Transl Med 2017; 9: eaai9048.

77 Bourgeois A, Lambert C, Habbout K, et al. FOXM1 promotes pulmonary artery smooth muscle cell expansion in pulmonary arterial hypertension. J Mol Med 2018; 96: 223-235.

78 Kudryashova TV, Goncharov DA, Pena A, et al. HIPPO-integrin-linked kinase cross-talk controls self-sustaining proliferation and survival in pulmonary hypertension. Am J Respir Crit Care Med 2016; 194: 866-877.

79 Vattulainen-Collanus S, Akinrinade $\mathrm{O}, \mathrm{Li} \mathrm{M}$, et al. Loss of PPARgamma in endothelial cells leads to impaired angiogenesis. J Cell Sci 2016; 129: 693-705.

80 Bertero T, Oldham WM, Cottrill KA, et al. Vascular stiffness mechanoactivates YAP/TAZ-dependent glutaminolysis to drive pulmonary hypertension. J Clin Invest 2016; 126: 3313-3335.

81 Zhao L, Oliver E, Maratou $\mathrm{K}$, et al. The zinc transporter ZIP12 regulates the pulmonary vascular response to chronic hypoxia. Nature 2015; 524: 356-360.

82 Boucherat O, Chabot S, Paulin R, et al. HDAC6: a novel histone deacetylase implicated in pulmonary arterial hypertension. Sci Rep 2017; 7: 4546.

83 Paulin R, Dromparis P, Sutendra G, et al. Sirtuin 3 deficiency is associated with inhibited mitochondrial function and pulmonary arterial hypertension in rodents and humans. Cell Metab 2014; 20: 827-839.

84 Michelakis ED, Gurtu V, Webster L, et al. Inhibition of pyruvate dehydrogenase kinase improves pulmonary arterial hypertension in genetically susceptible patients. Sci Transl Med 2017; 9: eaao4583.

85 Boucherat O, Peterlini T, Bourgeois A, et al. Mitochondrial HSP90 accumulation promotes vascular remodeling in pulmonary arterial hypertension. Am J Respir Crit Care Med 2018; 198: 90-103.

86 Bonnet S, Provencher S, Guignabert C, et al. Translating research into improved patient care in pulmonary arterial hypertension. Am J Respir Crit Care Med 2017; 195: 583-595.

87 Bonniaud P, Fabre A, Frossard N, et al. Optimising experimental research in respiratory diseases: an ERS statement. Eur Respir J 2018; 51: 1702133

88 Provencher S, Archer SL, Ramirez FD, et al. Standards and methodological rigor in pulmonary arterial hypertension preclinical and translational research. Circ Res 2018; 122: 1021-1032. 